\title{
Multiscale Simulation of Injection Molding of Parts with Low Aspect Ratio Microfeatures
}

\begin{abstract}
This article presents a simulation study of the injection molding of a macroscopic part with microfeatures; an optical grating with amplitude $0.6 \mu \mathrm{m}$ and period $3 \mu \mathrm{m}$. The aim of the simulations is to develop a procedure capable of predicting the replication of optical gratings based on the injection molding processing parameters. Three coupled simulations are performed. 1.) A macroscopic simulation of the flow in the part without microfeatures. 2.) A microscale flow simulation in a domain around a single microfeature using the pressure from the macroscopic simulation as input. 3.) A thermal simulation of the mold wall coupled to the microscale simulation.

In the simulations the polymer melt is treated as a generalized Newtonian fluid. Shear viscosity and thermal properties were characterized as part of the study. Other physical effects such as wall adhesion and elasticity are discussed, but not included in the present model.

The simulation results agree well with the experimental replication data, and correctly describe how small changes in the mold temperature can lead to large changes in the replication.
\end{abstract}

\section{Introduction}

Well-known examples of commercial polymer components with microfeatures include optical storage media and lightguide plates for LCD displays. Microfeatured polymer components are also key elements in many emerging technologies, such as labon-a-chip devices and optical sensors. Surfaces patterned with a combination of micro and nano features have also been demonstrated, altering optical characteristics (Saarikoski et al., 2009) and wetting properties (Puukilainen et al., 2007).

Producing microfeatured parts requires sharp definition of e.g. channels for microfluidics and gratings for diffractive optics. The aim of the present simulation study is to capture the observations in a previous experimental study in which a part with a micrometer scaled grating was injection molded. (Tofteberg et al., 2008). The part is illustrated in Fig. 1. The essential feature of this part is an optical grating with wavelength $3 \mu \mathrm{m}$ and peak to valley distance $0.6 \mu \mathrm{m}$. It has been molded with different amorphous polymers. In the present work only parts molded with a cyclic olefin copolymer (COC) is considered. COC has, because of its biocompatibility and good water barrier properties (Shin et al., 2005) become popular for producing labon-a-chip devices and has optical properties that make it attractive in for example waveguides (Khanarian and Celanese, 2001). In the experimental study (Tofteberg et al., 2008), the injection velocity and the mold temperature were varied, while keeping all other settings constant. After production, the degree of replication of the grating was characterized and it varied greatly with processing settings. Even small changes in the mold temperature gave large changes in the replication quality.

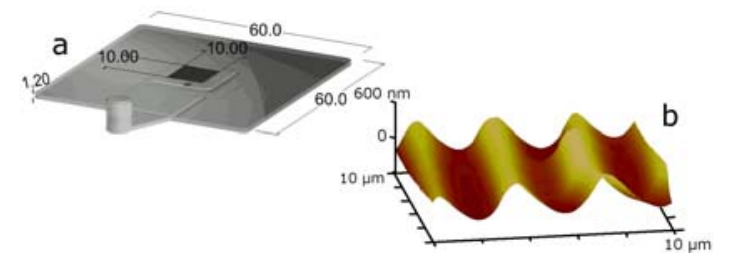

Fig. 1 a) Drawing of the injection molded part with dimensions $[\mathrm{mm}]$. The optical grating is located in the central dark square. b) Topography of the microfeatures (peak to valley distance $0.6 \mu \mathrm{m}$, period $3 \mu \mathrm{m})$ measured on an injection molded part using atomic force microscopy.

Microfeatures with low aspect ratio can be replicated well using conventional polymer processing techniques, such as injection molding and hot embossing. Consider for example the large scale production of CDs, DVDs and Blu-ray Discs which all have features in the sub micrometer range. Replication of features down to $10 \mathrm{~nm}$ has also been reported (Gadegaard et al., 2003). However, the detailed understanding on how such features form is limited. Their formation occurs under rapid cooling as the hot polymer melt makes contact with the mold wall. The temperature difference between the two is typically more than $100^{\circ} \mathrm{C}$, and the cooling rate of the polymer in the microfeatures can exceed $10.000 \mathrm{~K} / \mathrm{s}$ as will be shown. 


\subsection{Relevant physics on the microscale}

When the features are small there are several effects that might influence the filling, some of which are not necessarily important on the macroscale. These effects include wall adhesion, surface tension, wall slip and size dependent viscosity. The main reason for their increased importance on the microscale is that the surface to volume ratio is larger, making surface effects relatively more important than bulk properties. Yao and Kim (Yao and Kim, 2004) described several of these effects, their relevance to injection molding and how they scale with the size of the system.

One problem with surface effects is that they are more difficult to characterize than bulk properties. Equilibrium bulk properties will in general be given if the material and the thermodynamic state are known. Surface properties on the other hand depend on at least two materials, the thermodynamic state and in some cases also the size scale at which we observe the interface. One relevant example will be given to illustrate the last point. Consider two pieces of metal at different temperatures in contact. Heat will flow from the hotter piece to the colder. At the interface between the two there will be heat flow $q$ depending on the temperature difference $\Delta T$ between two materials and the apparent contact area $A_{\text {macro. }}$. The heat transfer coefficient can be defined as

$h_{\text {macro }}=\frac{q}{\Delta T \cdot A_{\text {macro }}}$

If the two materials are studied under a microscope, it will become clear that the metal surfaces are rough. The contact area at this resolution $A_{\text {micro }}$ is apparently smaller than the contact area on the macroscale. The heat flow and the temperature difference are still the same, which means that the microscopic heat transfer coefficient must be larger than the macroscopic one.

Specific microscale effects are so far not included in the simulations. The present focus is on coupling macroscale with microscale, and including the heating of the mold wall by the polymer. Nevertheless, it might be necessary to include microscale effects in future studies and their importance are estimated in the discussion section of this paper.

The high cooling rates close to the wall may influence both the rheology and the thermal properties of the melt. This is not directly a microscale phenomenon, but occurs because the microfeatures form in a very thin layer of the polymer in immediate contact with the cold mold wall. With a high cooling rate the glass transition temperature $\left(T_{g}\right)$ is shifted to higher temperatures than in measurements performed at low cooling rates, such as differential scanning calorimetry (DSC).

\subsection{Previous simulation studies}

For the part illustrated in Fig. 1 the ratio of part volume (a few $\mathrm{cm}^{3}$ ) to microfeature volume $\left(\sim \mu \mathrm{m}^{3}\right)$ is on the order of $10^{12}$. To perform a filling simulation using a uniform mesh will clearly be unsuitable as the number of elements will be too large to handle. Using a mesh which is finer in the vicinity of the microfeature will also lead to problems, as the time steps needed to describe the filling of the grating must be much shorter than the ones needed to describe the filling of the main geometry.

Several authors have proposed using a coupled simulation to solve this problem. A conventional injection molding filling simulation is performed to predict the filling of the main geometry without the microfeatures. Then a subset of the data from the macroscale simulation is used as input to a local simulation on the microscale. (Eriksson and Rasmussen, 2005, Kim and Turng, 2006, Yu et al., 2004) It is assumed that the microfeatures are so small that they exert only minor influence on the flow and the temperature field in the main geometry, and there is only a one way coupling from macroscale to microscale.

Eriksson and Rasmussen performed viscoelastic, isothermal simulations of the filling of micro ribs (width $22 \mu \mathrm{m}$ ) using a dual domain procedure (Eriksson and Rasmussen, 2005). The whole deformation history in the macroscopic simulation was used as an initial condition and the full stress tensor was employed as a boundary condition in the microscale simulation. An experimental setup was designed to provide an isothermal flow, terminated instantly by abrupt freezing of the polymer at a controlled time. These simulations gave insight to the filling process at isothermal conditions and showed quantitative agreement with the experimental filling lengths. The simulations indicated that the state of planar stretch as the polymer makes contact with the mold wall influences the filling of microfeatures. Isothermal simulations do, however, not describe the injection molding process, in which the rapid cooling of the polymer near the wall is an essential process determining the replication of microfeatures.

Kim and Turng (Kim and Turng, 2006) performed three-dimensional finite element filling simulations of an injection molded microfeature (rectangular plate with thickness $200 \mu \mathrm{m}$ and length $800 \mu \mathrm{m}$ ) on a flat plate using a viscous model for the melt viscosity. At the inlet to the microfeature they prescribed the pressure and the temperature from a separate filling simulation of the base plate. Their findings indicated that with a high injection velocity the heat transfer coefficient in the simulations had to be increased to explain the experimental data. 
Young gave an analytical approximation to the filling of microscale cylinders on a base geometry (Young, 2007). By neglecting the fountain flow effect in the filling of the main geometry and assuming a generalized Newtonian model for the rheology of the melt, an analytical expression for the cavity pressure behind the flow front was derived. This pressure and the initial melt temperature were used as boundary conditions for the microscale problem.

As described in a recent review on rapid thermal cycling of injection molds (Yao et al., 2008), controlling the mold surface temperature is the key to the replication of microfeatures. The surface temperature will not be constant during filling but increase as heat flows from the hot polymer to the mold. However, because the mold material has a much higher thermal conductivity than the polymer, the interface temperature will be closer to the initial mold temperature than the initial polymer temperature. This temperature rise is usually neglected when simulating the filling phase in conventional injection molding. The increase in mold temperature was estimated by Kim et al. (Kim et al., 2004) in a thermal simulation of an insulated mold coupled with an a simulation of the mold filling. Their simulations showed that the mold surface temperature could be kept higher for a longer time by insulating the cavity and it was argued that this would enhance the replication of microfeatures. The improved replication was also verified experimentally.

In the present article the coupled simulations on the macro and micro scales are combined with a thermal simulation of the mold temperature.

\section{Experimental determination of material parameters}

The polymer used in this study was a low viscosity cyclic olefin copolymer (COC), a copolymer of norbornene and ethylene (Topas 5013S-04 from Topas Advanced Polymers). It has a glass transition temperature $T_{\mathrm{g}}$ of $130{ }^{\circ} \mathrm{C}$ measured using differential scanning calorimetry at a cooling rate of $10^{\circ} \mathrm{C} / \mathrm{s}$. According to the datasheet, the temperature of the injected melt is recommended to be in the range 240$300{ }^{\circ} \mathrm{C}$, and mold temperatures up to $T_{\mathrm{g}}-10^{\circ} \mathrm{C}$ are recommended for products requiring high surface quality (even higher mold temperatures have been used during filling in variotherm processes).

\subsection{Rheology}

Since it is important to have a good description of the temperature dependence of the viscosity from the initial melt temperature down to the glass transition, it was seen necessary to perform rheological measurements as part of the study, and not only rely on available data, which was capillary rheometry data obtained at typical melt temperatures $\left(240-300^{\circ} \mathrm{C}\right)$ and high shear rates.
The complex viscosity $\eta^{*}$ was measured using dynamic rheometry. All measurements were performed with a Physica MCR 300 rheometer using a $25 \mathrm{~mm}$ diameter parallel plate fixture. Frequency sweeps were performed with angular frequency $\omega$ in the interval 0.1 to $500 \mathrm{~s}^{-1}$ at temperatures in the range $145-250{ }^{\circ} \mathrm{C}$. In addition, the zero-shear viscosity $\eta_{0}$ was measured at different temperatures in creep tests using the same rheometer.

The Cox-Merz rule has been shown to be valid experimentally for many rheologically simple materials

$$
\eta(\dot{\gamma})=\left|\eta^{*}(\omega)\right|_{\omega=\dot{\gamma}}
$$

The present COC grade is thermorheologically simple (Blochowiak et al., 2006) and we assume that the CoxMerz relation is valid.

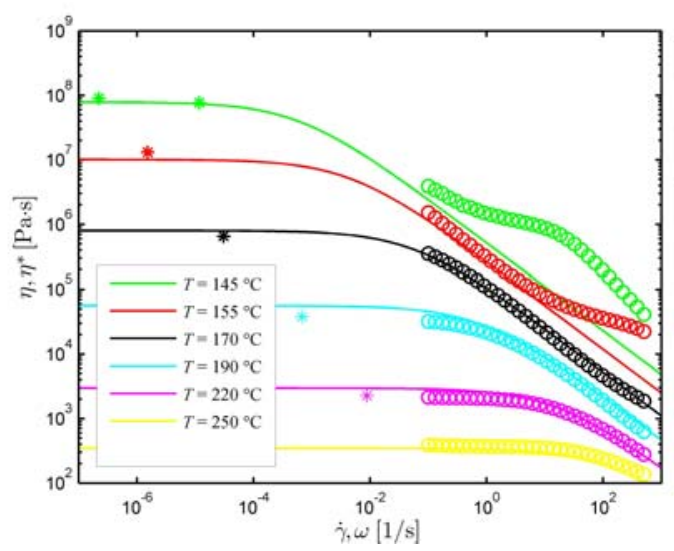

Fig. 2 Shear viscosity as a function of shear rate and temperature. Circles: Measured complex viscosity. Asterisks: Measured zero-shear viscosity. Solid lines: Fitted Cross-WLF model.

The Cross-WLF model was chosen to describe the rheology of the polymer melt

$$
\eta(T, \dot{\gamma})=\frac{\eta_{0}(T)}{1+\left(\frac{\eta_{0}(T) \dot{\gamma}}{\tau}\right)^{1-n}}
$$

$\log _{10} \frac{\eta_{0}(T)}{\eta_{0}\left(T_{\text {ref }}\right)}=\frac{-C_{1}\left(T-T_{\text {ref }}\right)}{C_{2}+\left(T-T_{\text {ref }}\right)}$

The Cross-WLF parameters were fitted to the experimental data as can be seen in Fig. 2. With $T_{\text {ref }}=145^{\circ} \mathrm{C}$, the coefficients are $\eta_{0}\left(T_{\text {ref }}\right)=7.96 \cdot 10^{7} \mathrm{~Pa} \cdot \mathrm{s}, \quad C_{1}=11.3, \quad C_{2}=117.4 \mathrm{~K}$, $n=0.32$ and $\tau=47.6 \mathrm{kPa}$.

The measured viscosity, spanning six decades, is well described by this model. However, it can be seen that for frequencies above $\sim 10 \mathrm{~s}^{-1}$ at $155^{\circ} \mathrm{C}$, the viscosity is higher than what is expected from the Cross-WLF model. The same is the case at other temperatures; the 
threshold frequency decreases with decreasing temperature. This increase in the viscosity is the glass transition and the frequency shift is an example of the time-temperature superposition principle. Finally, it can be mentioned that our viscosity measurements at high temperatures agree with data by others at typical processing temperatures $\left(240-300^{\circ} \mathrm{C}\right)$ for this material.

In the simulations it is clear that the Cross-WLF model does not give a good description at low temperatures. Hence, a no-flow temperature close to $T_{g}$ was designated. When the flow front reaches this temperature, the flow front position is recorded and used to calculate the final degree of replication.

For many amorphous polymers, the apparent $T_{\mathrm{g}}$ will increase by approximately $3{ }^{\circ} \mathrm{C}$ per decade that the timescale of the system is changed (Ferry, 1980). In the present work, the cooling rate as the polymer passes through $T_{g}$ is between 100 and $10.000{ }^{\circ} \mathrm{C} / \mathrm{s}$ depending on the processing parameters. This is equivalent to between three and five decades faster than the DSC measurements. Hence, the expected shift in apparent $T_{g}$ is in the range $9-15^{\circ} \mathrm{C}$. It will be shown in the simulations that the choice of no-flow temperature is of minor importance at the highest cooling rates. Therefore, in this study we chose a no-flow temperature of $140{ }^{\circ} \mathrm{C}, 10^{\circ} \mathrm{C}$ above the $T_{\mathrm{g}}$ obtained by DSC.

The reason for using a generalized Newtonian model to describe the melt, despite its limitations, is because it greatly simplifies the numerical simulations compared to using a viscoelastic model. Generalized Newtonian models are available in most CFD codes and commercial software dedicated to injection molding presently only use viscous models in the filling stage. It is therefore interesting to see how far it is possible to come with such an approximation.

\subsection{Thermal properties}

The thermal diffusivity of the polymer was measured using a laser flash diffusivity apparatus (LFA 457 MicroFlash from NETZSCH). The specific heat was measured using a differential scanning calorimeter (DSC 204 F1 Phoenix from NETZSCH). When calculating the thermal conductivity a constant melt density $\rho_{\mathrm{COC}}=1003 \mathrm{~kg} / \mathrm{m}^{3}$ was used. This approximation was also made in the simulations. The measured thermal properties are summarized in Fig. 3. Linear interpolation of these data was employed in the simulations. Any pressure or cooling rate dependence of the variables was neglected.
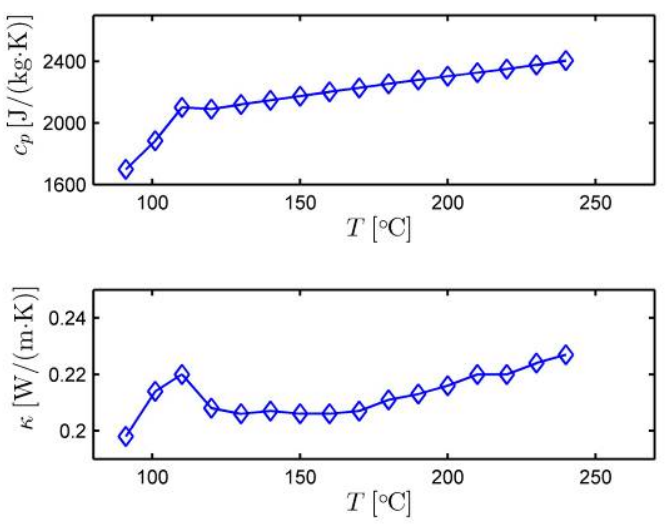

Fig. 3 Heat capacity $\left(c_{\mathrm{p}}\right)$ and thermal conductivity ( $\kappa$ ) for the COC polymer (symbols). In the simulations, linear interpolation of the data was used (solid lines).

Air is trapped inside the microfeatures as the polymer enters, see Fig. 4. The air is treated explicitly in the simulations, with the following properties: Thermal conductivity $\kappa_{\text {Air }}=0.0261 \mathrm{~W} / \mathrm{mK}$, heat capacity $c_{\mathrm{p}, \mathrm{Air}}=1004 \mathrm{~J} / \mathrm{kgK}$ and viscosity $\eta_{\text {Air }}=2 \cdot 10^{-5} \mathrm{~Pa} \cdot \mathrm{s}$. Effects of pressure and temperature on these parameters are neglected. The air density $\rho_{\text {Air }}$ is given from an ideal gas expression

$\rho_{\text {Air }}=\frac{p M_{\mathrm{w}}}{R T}$,

where $p$ is the pressure, $T$ the temperature, $R=8.31 \mathrm{~J} / \mathrm{molK}$ the universal gas constant and $M_{\mathrm{w}}=29 \mathrm{~g} / \mathrm{mol}$ the molecular weight. Dissolution of air in the polymer is neglected.

The thermal properties of the mold materials are given in Table 1. Temperature dependence is neglected.

Table 1 Properties of mold and mold insert materials used in thermal simulations.

\begin{tabular}{|l|c|c|c|c|}
\hline Material & $\begin{array}{c}\rho \\
{\left[\mathrm{kg} / \mathrm{m}^{3}\right]}\end{array}$ & $\begin{array}{c}\kappa \\
{[\mathrm{W} / \mathrm{mK}]}\end{array}$ & $\begin{array}{c}c_{\mathrm{p}} \\
{[\mathrm{J} / \mathrm{kgK}]}\end{array}$ & $\begin{array}{c}\text { Thickness } \\
{[\mathrm{mm}]}\end{array}$ \\
\hline Nickel & 8800 & 91 & 440 & 0.3 \\
\hline BeCu & 8770 & 245 & 430 & 10 \\
\hline Steel & 7800 & 27.5 & 460 & 23 \\
\hline
\end{tabular}

\section{Simulation procedure}

Three coupled simulations are performed. First, the filling of the macroscopic geometry is simulated. The pressure from this simulation is then used as a boundary condition in the microscale flow simulation. The third simulation is a one-dimensional simulation of the temperature evolution in the mold wall, coupled with the microscale flow simulation.

\subsection{Macroscale flow simulation}

The macroscale simulation is performed in a commercial injection molding simulation software 
(Moldflow Plastics Insight (MPI) 6.1, 2007). Since the geometry is essentially flat as seen in Fig. 1a; a $2.5 \mathrm{D}$ representation was used in the macroscale simulation. The injection velocity and the melt temperature are specified as input and only the filling phase of the injection molding cycle is considered. The purpose of the macroscale simulation is to obtain the cavity pressure for the microscale simulation and this is the only information passed from the macroscale to the microscale. This pressure was also measured using a pressure sensor mounted in the mold half opposite the microfeatures, and the measurements showed good agreement with the simulated pressure as was shown earlier (Tofteberg et al., 2008).

\subsection{Microscale flow simulation}

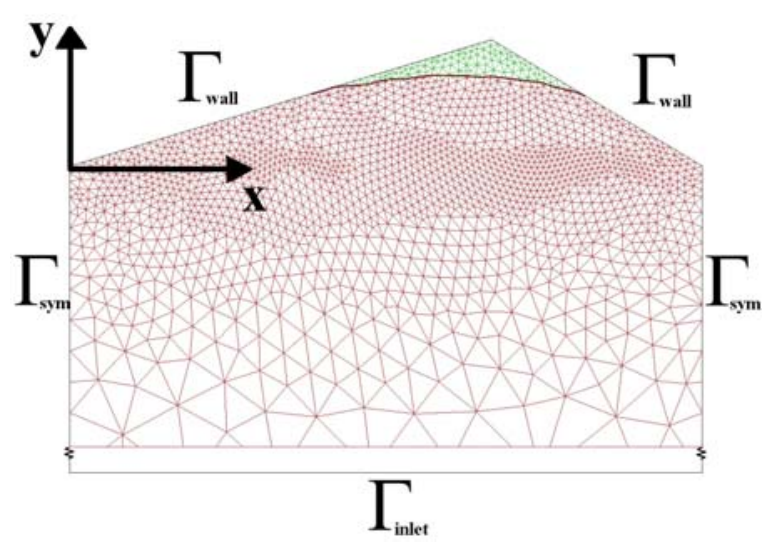

Fig. 4 Illustration of the flow front during a microscale flow simulation. The mesh is colored red in the region where the polymer is present and green in the air region. The boundary conditions are given in the main text.

An illustration of the situation when the polymer melt hits the microstructured nickel shim (mold insert) is illustrated in Fig. 5. Behind the shim are two more metal mold components. There is no change in the microfeature geometry in the out of plane direction. Hence, 2D flow was assumed in the microscale simulation.

It can be shown analytically that when a Newtonian fluid fills the gap between two parallel plates, the flow front will take a shape very close to a semi-circle (Gramberg et al., 2004). Since the period of the optical grating $(3 \mu \mathrm{m})$ is very small compared to the thickness of the base geometry $(1.2 \mathrm{~mm})$, the flow front will be essentially flat as it makes contact with the wall. Such a flat flow front (at $y=0$ in Fig. 4) is used as the initial condition in the microscale simulation. The filling of the microfeatures is treated essentially as a compression molding problem, but instead of prescribing the movement of a mold/master, the cavity pressure from the macroscale simulation is used to force the polymer into the microfeatures.

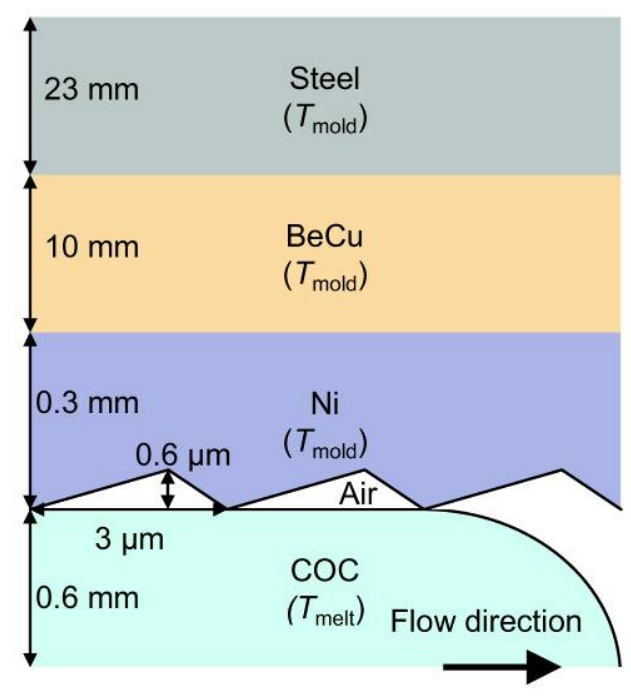

Fig. 5 Sketch of the polymer melt hitting the microfeatures at the wall. Note that the sketch is not to scale. The temperatures indicated in parentheses are the initial temperatures in the simulations.

A general fluid dynamics (CFD) solver was used (ANSYS CFX-11.0, 2007) to solve the microscale problem. The code is based on the finite volume method and has previously been used for 3D mold filling simulations (Vahlund, 2003).

\subsubsection{Governing equations}

The two-phase (polymer-air) flow problem is solved using what in the software is called a homogenous model. This means that the two phases share common velocity, pressure and temperature fields and the physical properties of the fluid depend on a phase variable. The equation of conservation of momentum reads

$\frac{\partial}{\partial t}(\rho \mathbf{U})+\nabla \cdot(\rho \mathbf{U} \otimes \mathbf{U})=-\nabla p+\nabla \cdot\left(\eta\left[\nabla \mathbf{U}+(\nabla \mathbf{U})^{\mathbf{T}}\right]\right)$,

where $\mathbf{U}$ is the velocity vector, $\cdot$ is the inner product of two vectors, $\otimes$ is the outer product and $p$ is the pressure. The density $\rho$ and the viscosity $\eta$ are linear interpolations over a composition variable $r$.

$$
\begin{aligned}
& \rho=r_{\text {Air }} \rho_{\text {Air }}+r_{\mathrm{COC}} \rho_{\mathrm{COC}} \\
& \eta=r_{\text {Air }} \eta_{\text {Air }}+r_{\mathrm{COC}} \eta_{\mathrm{COC}}
\end{aligned}
$$

The volume fractions take values between zero and one and sum to unity.

$$
r_{\mathrm{Air}}+r_{\mathrm{COC}}=1
$$

Initially there is a sharp step in the volume fraction variable. During the simulation the interface region diffuses out to a width of approximately two mesh elements. This description is not meant to capture the 
physics of the interface. It is a construction to treat a multiphase problem with a moving interface on a fixed grid. The equation of continuity for each phase reads

$\frac{\partial}{\partial t}\left(r_{\alpha} \rho_{\alpha}\right)+\nabla \cdot\left(r_{\alpha} \rho_{\alpha} \mathbf{U}\right)=0$

where $\alpha$ designates the phase, either Air or COC. The equation for conservation of energy includes heat conduction, convection and viscous dissipation;

$\frac{\partial}{\partial t}(\rho e)+\nabla \cdot(\rho \mathbf{U} e)=\nabla \cdot(\kappa \nabla T)+\boldsymbol{\tau}: \nabla \mathbf{U}$,

where $e$ is the internal energy per unit mass, $T$ is the temperature, $\kappa$ is the thermal conductivity and $\tau$ is the stress tensor. Both the internal energy and the thermal conductivity are taken as linear interpolations over $r_{\alpha}$. The compression work of the gas phase is neglected in the energy equation. This work is not large and can not be implemented in the present release of the software.

\subsubsection{Boundary conditions}

At the mold wall, a no-slip condition is used for the velocity and a heat transfer coefficient $h$ is used for the thermal boundary condition where the polymer is in contact with the wall.

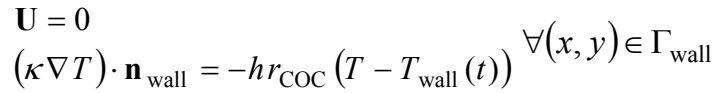

$\mathbf{n}_{\text {wall }}$ is the outward pointing wall normal vector and $T_{\text {wall }}$ is the temperature of the mold wall. This wall temperature is coupled with a mold wall temperature simulation as described in the next section. The heat transfer coefficient was given by the expression below (discussed later)

$$
h=30.000 \mathrm{~W} / \mathrm{m}^{2} \mathrm{~K}
$$

On the microscale, only one period of the repeating grating is included in the simulation as shown in Fig. 4. To simulate the effect of a repeating grating, symmetry conditions were applied on the two boundaries labelled $\Gamma_{\text {sym }}$ in Fig. 4. This implies zero velocity across the boundary, no shear stress tangential to the surface and zero heat flux.

$$
\begin{aligned}
\mathbf{U} \cdot \mathbf{n}_{\mathrm{sym}} & =0 \\
\boldsymbol{\tau}_{\mathrm{sym}} & =0 \quad \forall(x, y) \in \Gamma_{\mathrm{sym}} \\
(\kappa \nabla T) \cdot \mathbf{n}_{\mathrm{sym}} & =0
\end{aligned}
$$

where $\mathbf{n}_{\text {sym }}$ is the outward pointing normal vector and $\boldsymbol{\tau}_{\text {sym }}$ is the shear stress at the boundary. An attempt was made to use periodic boundary conditions. The results were almost identical, as both the flow and the heat conduction through the boundary were very small, but the numerical stability was not good.
At the inlet labeled $\Gamma_{\text {inlet }}$ in Fig. 4, the pressure $p(t)$ is taken from the macroscopic simulation. In other models for flow into microfeatures (Kim and Turng, 2006, Young, 2007) a pressure boundary condition is applied directly at the inlet to the microfeature ( $y=0$ in Fig. 4). This is similar to our approach, but we apply the pressure condition further away from the inlet. By doing this, the pressure loss occurring close to the entrance to the microfeature is included.

The inlet location is chosen far from the mold wall, so that the temperature here can be assumed to remain constant at $T_{\text {melt }}$ during the simulation. The location was chosen to be $0.6 \mathrm{~mm}$ from the wall, i.e. at the center of the macroscale geometry.

$p=p(t) \quad \forall(x, y) \in \Gamma_{\text {inlet }}$
$T=T_{\text {melt }}$

After simulating the flow, it was verified that only a thin layer close to the wall was cooled significantly. Only regions closer to the wall than $100 \mu \mathrm{m}$ were cooled by more than $1{ }^{\circ} \mathrm{C}$ and the assumption of a constant temperature at the inlet was found acceptable.

When the macroscopic geometry is completely filled, a constant holding pressure $(50 \mathrm{MPa})$ is prescribed. However, at the time the holding pressure was applied, the polymer had, reached its no-flow temperature (in all the cases investigated). Hence, the holding pressure did not influence the simulated replication of the microfeatures.

\subsubsection{Initial conditions}

The microscale simulation starts when the polymer melt makes contact with the wall at the location of the microfeature. Initially there is a sharp step in $r_{C O C}$ (Eq. 15, see Fig. 4 for the coordinate system), and the entire domain has a uniform temperature equal to the initial melt temperature and zero velocity.

$$
\begin{aligned}
r_{\mathrm{COC}}(t=0) & =\left\{\begin{array}{l}
1 \text { for } y<0 \\
0 \text { for } y>0
\end{array}\right. \\
T(t=0) & =T_{\text {melt }} \\
\mathbf{U}(t=0) & =0
\end{aligned}
$$


The reasoning for choosing $T_{\text {melt }}$ as the initial temperature is partly that we observed that the flow front temperature in the macroscale simulation changed by less than $1{ }^{\circ} \mathrm{C}$ during filling with these processing conditions. Also, the polymer making contact with the wall has been transported outwards from the middle of the macroscopic fountain flow, and it has not been in proximity with the cold wall before making contact with the microfeature.

\subsection{Mold wall thermal simulation}

A one-dimensional heat conduction problem was solved through the mold wall:

$\rho c_{p} \frac{\partial T}{\partial t}=\frac{\partial}{\partial y}\left(\kappa \frac{\partial T}{\partial y}\right)$

The boundary condition at $y=0$ is the heat flux from the microscale simulation.

$\left.\kappa \frac{\partial T}{\partial y}\right|_{y=0}=\Phi(t)$

where the heat flux is calculated at each time step as the integral of the heat flow over the mold wall divided by the area of the microfeature projected into the plane of the wall $L_{\mu}=3 \mu \mathrm{m}$.

$\Phi(t)=\frac{-1}{L_{\mu}} \int_{\Gamma_{\text {wall }}} h r_{\mathrm{COC}}\left(T-T_{\text {wall }}\right) \mathrm{d} \Gamma_{\text {wall }}$

The temperature at the location of the water cooling channels is assumed constant.

$T(y=33.3 \mathrm{~mm})=T_{\text {mold }}$

Initially the temperature of the entire domain is assumed to be uniform. The time variable is the same in the microscale and the mold wall thermal simulation.

$T(t=0)=T_{\text {mold }}$

This partial differential equation was solved using the function pdepe in Matlab (Matlab R2007a, 2007).

\subsection{Coupling microscale flow and thermal simulations}

The microscale simulation and the mold wall thermal simulation were solved using an iterative procedure; 1.) An initial time dependent guess was made for the mold wall temperature $T_{\text {wall }}(t)$ in Eq. 11. 2.) A full microscale simulation was carried out and the heat flux through the wall was calculated at each time step. 3.) This heat flux was integrated over the wall, projected onto the plane of the wall and used in Eq. 17 for a thermal simulation through the mold wall 4.) The temperature in the thermal simulation at $y=0$ was used as a new estimate for $T_{\text {wall }}$. Then step 2-4 was repeated until convergence. After two iterations the mold wall temperature had converged.

\section{Results and discussion}

\subsection{Definitions}

Macroscopic injection velocity $\left(v_{\text {macro }}\right)$ : The volumetric flow rate divided by the cross-sectional area of the part.

Microscale flow front position: The contour line with volume fraction $r_{\mathrm{COC}}=0.5$.

Microscale flow front temperature $\left(T_{\text {flow front }}\right)$ : The areaaverage temperature over the flow front. The temperature at the flow front is relatively uniform; the difference between the maximum and minimum is less than $1{ }^{\circ} \mathrm{C}$.

Degree of replication (DOR): The DOR is defined as in the experimental paper, and is based on the Fourier transform of the replicated grating (Tofteberg et al., 2008). It is found by first transforming the shape of the periodic grating into the frequency domain. The DOR is then the strength of the strongest frequency (the inverse of the period) relative to the strength of that frequency measured on the shim. The mathematical definition is given in the Appendix. This expression is used for the final DOR, as well as the DOR during filling of the microfeatures. The values obtained by this method correlate well with the peak to valley distance of the molded gratings. The reason for choosing this parameter in the frequency domain is that experimentally it is faster and more robust to measure this parameter than the individual peak heights of the microfeatures.

\subsection{Flow front advancement}

Fig. 6 shows the filling of the microfeature as a function of time for different mold temperatures and injection velocities. As the melt hits the wall, it has the initial temperature of $270{ }^{\circ} \mathrm{C}$. This means that the temperature difference in the wall temperature boundary condition (Eq. 11) is relatively insensitive to small differences in mold temperature. Hence, at a given injection velocity, the microfeature is initially filled at a rate independent of the mold temperature. After a few milliseconds the flow front has cooled enough for the flow to be is sensitive to differences in mold wall temperature, and the filling curves for different mold temperatures diverge.

When comparing the results for different injection velocities in Fig. 6, the filling curves are shifted to lower $t$ values when increasing the injection velocity. The initial advancement of the flow front is faster, the diverging behavior for different mold temperatures occur earlier and the no-flow temperatures are reached at an earlier time. These three phenomena are naturally linked to the faster buildup of pressure and the resulting reduction in thermal contact resistance. 

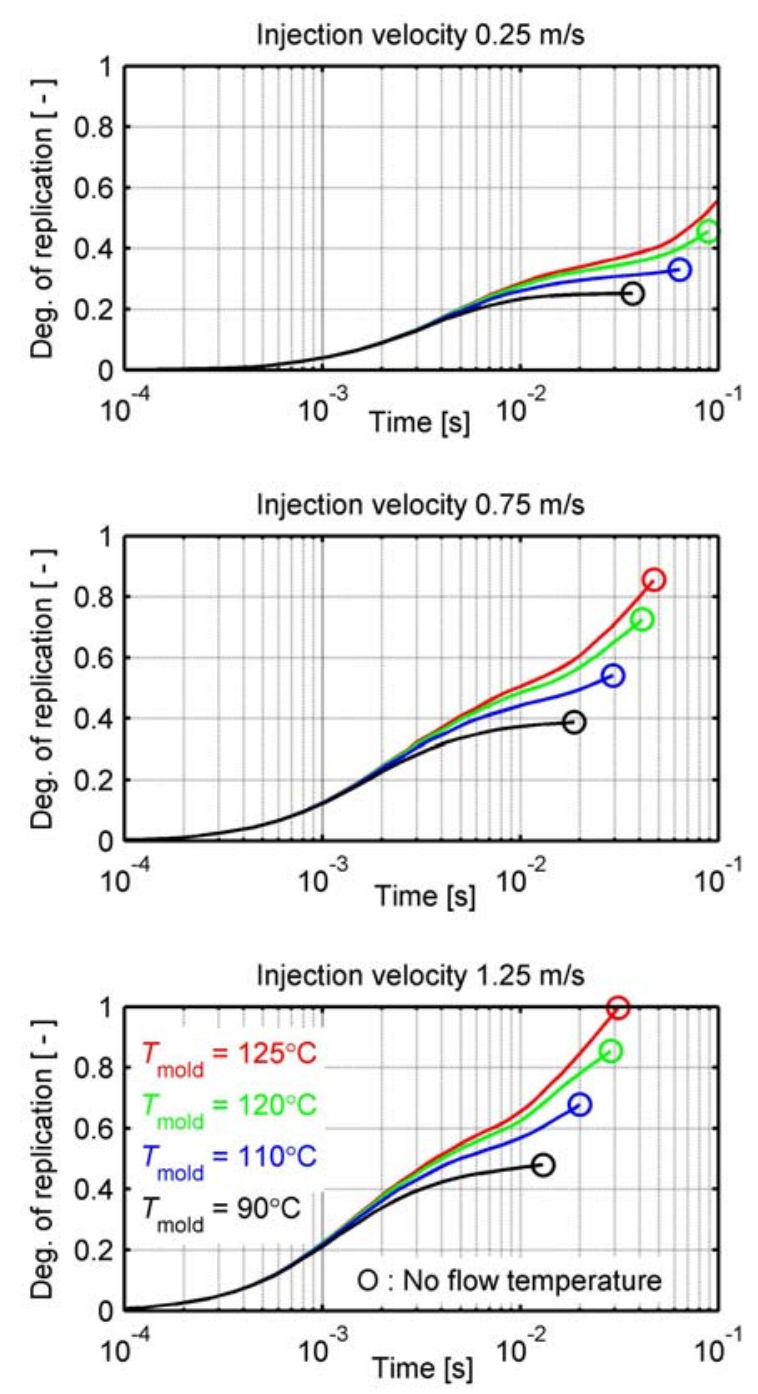

Fig. 6 Simulated degree of replication as function of time after first polymer/mold wall contact for different mold temperatures $T_{\text {mold }}$ and injection velocities. The circles indicate when the polymer has reached its no-flow temperature of $140^{\circ} \mathrm{C}$. See 4.1 for definitions.

This temperature effect is clearer when plotting the degree of replication as a function of the flow front temperature as seen in Fig. 7. In this figure time runs from left to right. The flow front temperature starts at $270{ }^{\circ} \mathrm{C}$ and as the polymer cools down it protrudes further into the microfeature until the no-flow temperature is reached. Consider the curves for a mold temperature of $90^{\circ} \mathrm{C}$. At least for low to medium injection velocities, these curves reach a plateau before the no-flow temperature is reached. Because of the high cooling rate, an effective no-flow temperature is reached at around $150{ }^{\circ} \mathrm{C}$.
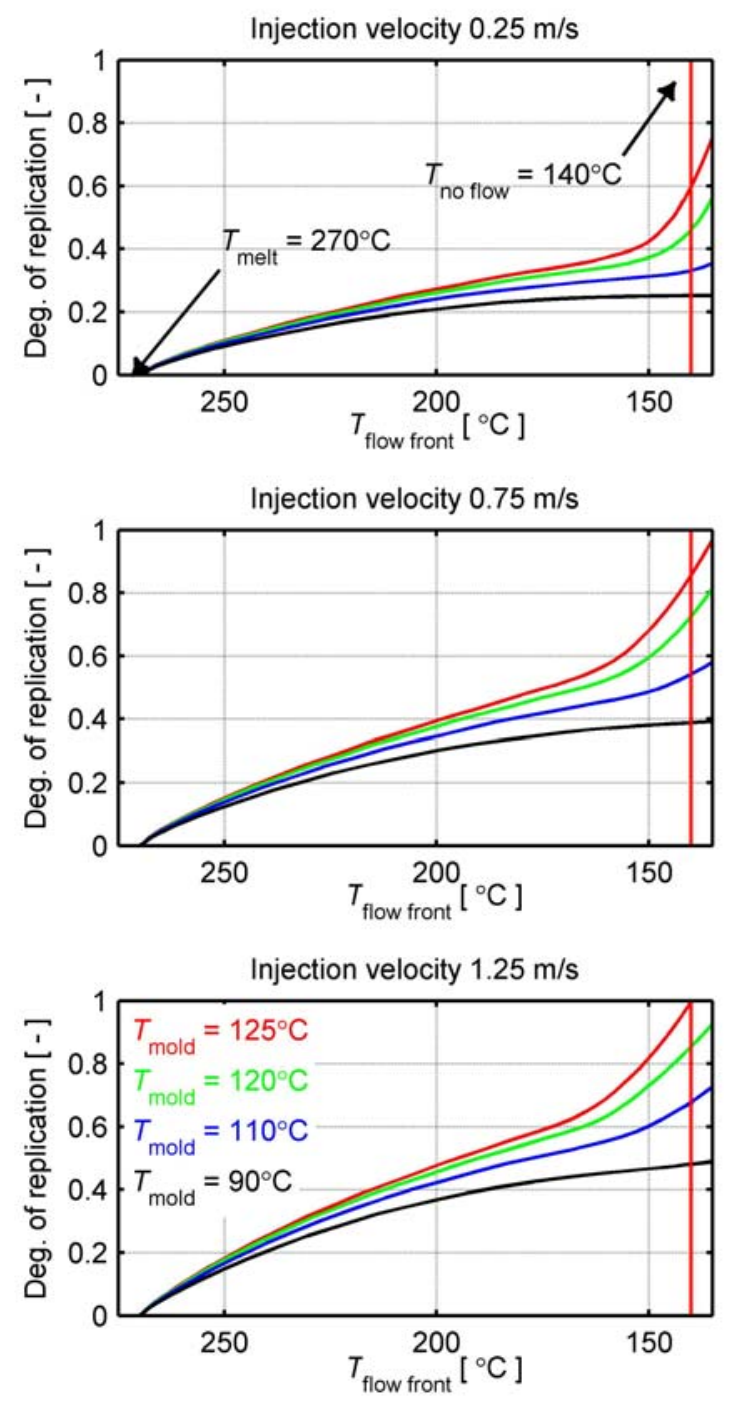

Fig. 7 Simulated degree of replication as function of flow front temperature for different mold temperatures $T_{\text {mold }}$ and injection velocities $v_{\text {macro }}$. See 4.1 for definitions. Time is increasing from left to right.

This behavior is not seen for higher mold temperatures. Most of the difference in replication between using a mold temperature of $125^{\circ} \mathrm{C}$ and $120^{\circ} \mathrm{C}$ is due to flow at temperatures between $140^{\circ} \mathrm{C}$ and $150^{\circ} \mathrm{C}$. As shown in Fig. 2, the rheology of the melt is not well modeled at these temperatures and caution should be made when interpreting the results.

Finally, the microscale flow front temperature as a function of time is shown in Fig. 8. It can be seen that the polymer is above the no-flow temperature $50 \%$ longer when using a mold temperature of $120{ }^{\circ} \mathrm{C}$ instead of $110^{\circ} \mathrm{C}$, with an injection velocity of $0.75 \mathrm{~m} / \mathrm{s}$. And as can be seen in Fig. 7 this leads to a significant improvement in replication. 

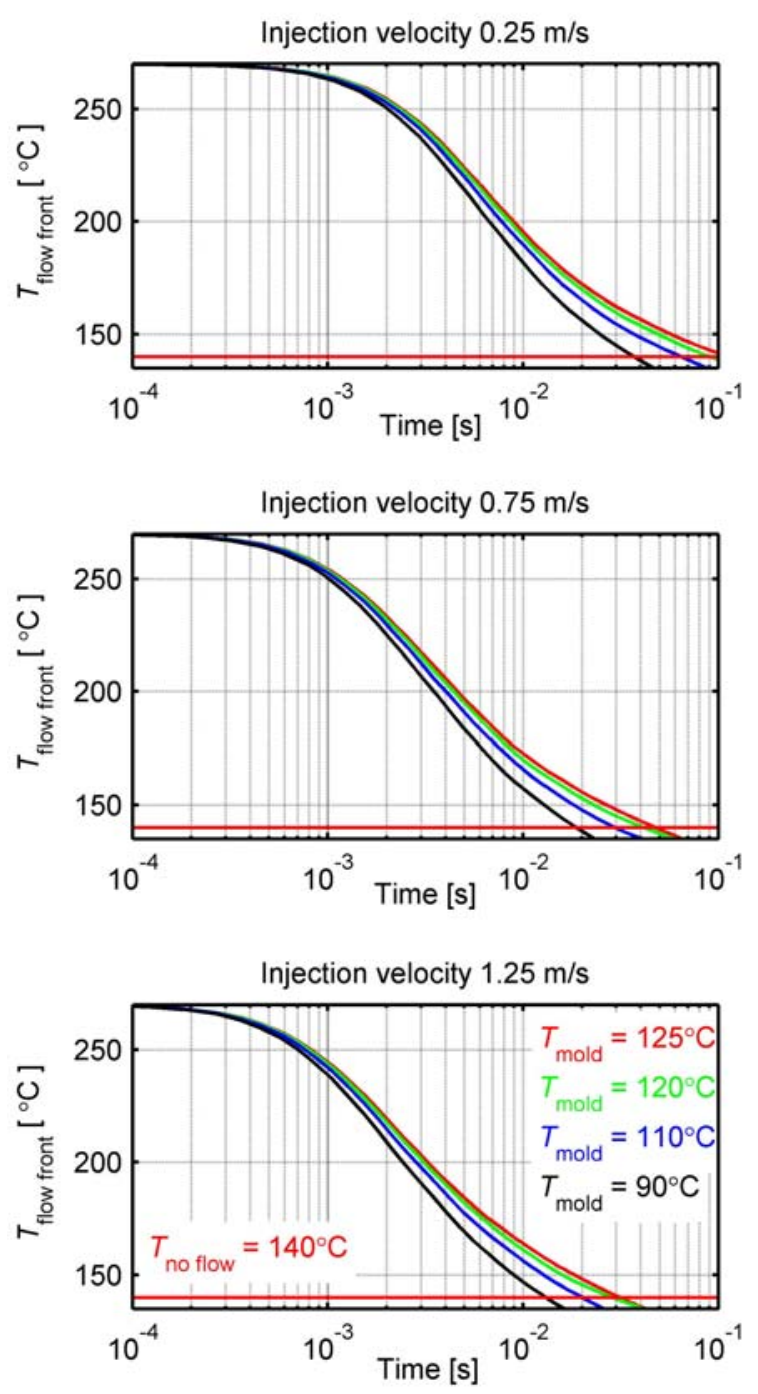

Fig. 8 The flow front temperature as a function of time after first polymer/mold wall contact at different mold temperatures $T_{\text {mold }}$ and injection velocities. See 4.1 for definitions.

\subsection{Comparison with experiments}

In the experimental study, parts with microfeatures were molded using different values for the mold temperature and injection velocity while keeping the holding pressure and all other processing settings constant. The replication was assessed using white light interferometer. The experimental replication is compared to the final degree of replication from simulations in Fig. 9. The overall agreement is good. It is seen, for example that the difference in replication when reducing the mold temperature from $125^{\circ} \mathrm{C}$ to $120{ }^{\circ} \mathrm{C}, 120^{\circ} \mathrm{C}$ to $110{ }^{\circ} \mathrm{C}$ and $110^{\circ} \mathrm{C}$ to $90{ }^{\circ} \mathrm{C}$ is approximately the same, even though the temperature drop is 5,10 and $20^{\circ} \mathrm{C}$ respectively. This increased sensitivity in replication with respect to mold temperature when approaching the glass transition temperature is correctly described by the simulations.

The sensitivity to injection velocity is somewhat over predicted in the simulations. The most noticeable disagreement is seen with the highest mold temperature $\left(125^{\circ} \mathrm{C}\right)$ and low injection velocities. Almost complete replication is observed experimentally, but not in the simulations. The replication being nearly independent of injection velocity at this mold temperature, as observed experimentally, indicates that the microfeatures might be filled by the holding pressure applied after the end of the filling phase. As the temperature drops below $T_{\mathrm{g}}$, the polymer can still be elastically deformed by the holding pressure. This deformation is then frozen in as the polymer cools further. Such an effect can not be captured with a viscous rheology model.

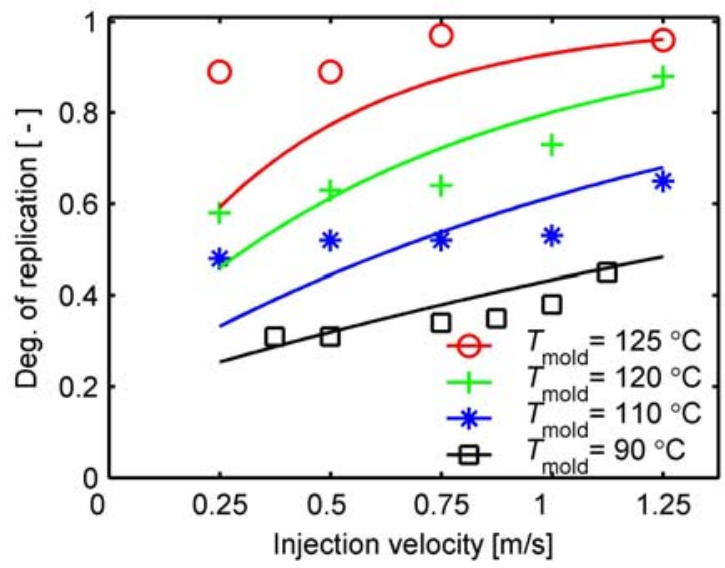

Fig. 9 Final degree of replication from simulations (solid lines) and experiments (symbols). The experimental

\subsection{Heating of the mold wall}

Fig. 10 shows a typical simulation of the mold surface (Nickel shim) temperature vs. time. In this example the initial mold temperature was $120^{\circ} \mathrm{C}$, but the observed temperature increase was very similar at other mold temperatures. After $0.01 \mathrm{~s}$, the surface temperature has increased by almost $4{ }^{\circ} \mathrm{C}$. This is compared to the ideal contact temperature between pure nickel and $\mathrm{COC}$ given by their relative effusivities $b$.

$T_{\text {contact }}=\frac{b_{1} T_{1}+b_{2} T_{2}}{b_{1}+b_{2}}, \quad b_{i}=\sqrt{\rho_{i} c_{p, i} \kappa_{i}}$

This ideal contact temperature is calculated by considering two semi-infinite one-dimensional bodies initially at temperatures $T_{1}$ and $T_{2}$. If the two bodies are brought into perfect thermal contact at $t=0$, the contact temperature at the surface of the two bodies will be given by 21 for all positive times.(Baehr and Stephan, 2004) In the real world, no true semi-infinite bodies exist, but the simplification can be a good approximation for short times. As long as the timescale 
is so short that all other thermal boundaries are not influenced by the contact boundary, a body can be considered semi-infinite. Relative effusivities have previously been used to estimate the wall surface temperature (Yoshii et al., 1998).

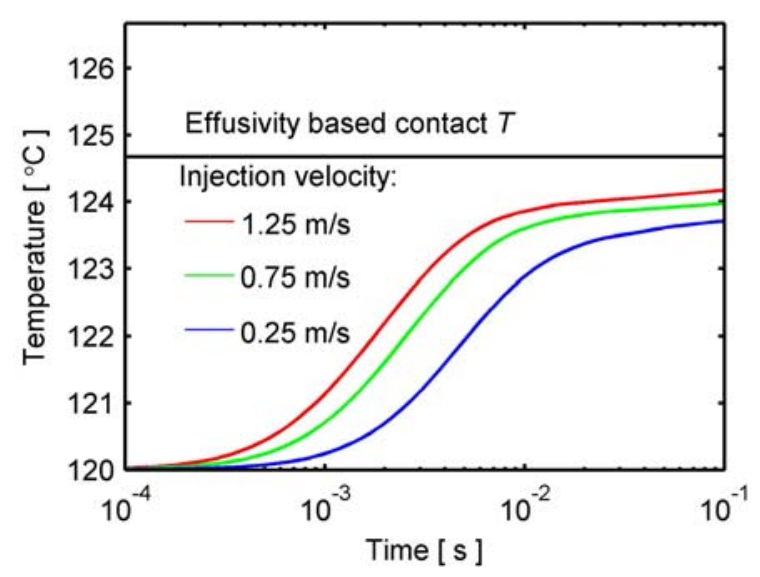

Fig. 10 Simulated surface temperature of the nickel shim as a function of time for different injection velocities. The horizontal line is the ideal contact temperature between $\mathrm{COC}$ and nickel based on effusivity values.

\section{Discussion}

\subsection{The heat transfer coefficient}

The simulation results are sensitive to the choice of heat transfer coefficient $(h)$ and the replication at all processing settings are reduced with an increasing value for $h$. The value in this study was chosen so that almost complete replication was achieved at a mold temperature of $125^{\circ} \mathrm{C}$ and injection velocity $1.25 \mathrm{~m} / \mathrm{s}$ as observed experimentally. Tuning the microscale heat transfer coefficient to match simulated data with experimental data is a procedure also used in other studies. (Kim and Turng, 2006, Yu et al., 2004). Changing the heat transfer coefficient will change the predicted replication, but the dependence on processing settings will still be similar.

When the heat transfer coefficient is chosen so that the experimental results and the simulated results agree, there is an apparent danger of camouflaging other relevant effects. If a relevant effect such as the wall adhesion is neglected, it can to a certain extent be compensated for by a change in the heat transfer coefficient

The value used in this work was relatively high compared to values used in conventional injection molding. Our rationale for this is that the apparent contact area on the microscale is smaller than on the macroscale. Another reason is that the heating of the mold was included in the present simulations. If not included, the temperature difference between mold and polymer would have been larger in the simulation than the actual value. This could to some extent be compensated for by using a lower heat transfer coefficient.

In previous simulation studies (Kim and Turng, 2006, Yu et al., 2004), it was found that a higher heat transfer coefficient was needed to explain replication data at higher injection velocities. A velocity dependent heat transfer coefficient was not introduced in the present study as we do not have a quantitative model to describe such a relationship on the microscale. However, it should be noted that a heat transfer coefficient increasing with injection velocity (or cavity pressure) would improve the agreement between simulations and experiments, by reducing the simulated increase in replication with increasing injection velocity.

\subsection{Wall adhesion}

We have not measured the adhesion energy between the polymer (COC) and the mold wall. However, the adhesion energy between several high density polyethylenes (HDPE) and steel is on the order of $\gamma=0.03 \mathrm{~J} / \mathrm{m}^{2}$ (Anastasiadis and Hatzikiriakos, 1998). Chemically, COC and HDPE are similar polymers, consisting only of carbon atoms saturated with hydrogen. The adhesion energy between COC and nickel is therefore believed to be of the same order of magnitude.

The work $W_{\text {pressure }}$ required to fill a microfeature at constant pressure $p$ is

$W_{\text {pressure }}=p \cdot V_{\text {micro }}$,

where $V_{\text {micro }}$ is the volume of the microfeature. The work required to fill the microfeature by wall adhesion alone is

$$
W_{\text {adhesion }}=\gamma \cdot A_{\text {contact }},
$$

where $A_{\text {contact }}$ is the contact area between the wall and the polymer. If Eq. 22 and 23 are assumed to be equal, a capillary pressure representing the same driving force as the wall adhesion can be calculated. Using $\gamma=0.03 \mathrm{~J} / \mathrm{m}^{2}$ and the present geometry, gives a capillary pressure of $0.1 \mathrm{MPa}$. This could be a significant contribution to the filling, the first milliseconds, especially for low injection velocities, when the cavity pressure increases slowly.

The effect of surface tension can also be evaluated by considering the dimensionless capillary number $\mathrm{Ca}$. It is defined as the ratio between viscous forces and surface tension.

$\mathrm{Ca}=\frac{\hat{U} \hat{\eta}}{\hat{\sigma}}$,

where $\hat{U}$ is a characteristic velocity, $\hat{\eta}$ a characteristic viscosity and $\hat{\sigma}$ a characteristic surface tension. We 
define the area average value over the flow front as the characteristic velocity and viscosity. The surface tension for different solid COC materials has been measured to be in the range $0.035-0.045 \mathrm{~N} / \mathrm{m}$ (Shin et al., 2005). In the present simulations $\mathrm{Ca}<1$ for $\mathrm{t}<1 \mathrm{~ms}$. This indicates a flow where capillary action is important. The capillary number then increases rapidly and after around 10 milliseconds $\mathrm{Ca}>100$ and surface tension can be neglected.

\subsection{Viscous dissipation}

The relative importance of viscous dissipation to wall heat transfer can be evaluated using the Brinkman number

$\mathrm{Br}=\frac{\hat{U}^{2} \hat{\eta}}{\kappa \Delta T}$

where $\Delta T$ is the temperature difference between the mold wall and the polymer. For all parameters the characteristic values used are the area average over the flow front. During the present microscale simulations, the Brinkman number is always below $10^{-3}$ and viscous dissipation in the microfeature can be disregarded.

\subsection{Conduction and convection}

The relative importance of heat conduction and heat convection can be evaluated using the Peclet number.

$$
\mathrm{Pe}=\frac{\hat{U} \hat{h} \rho c_{p}}{\kappa}
$$

The characteristic length $\hat{h}$ is taken as the height of the microfeature $(0.6 \mu \mathrm{m})$ and all other properties are evaluated at the flow front as area averages. As the velocities in the present simulations are small, the Fourier number is initially below $10^{-2}$ and decreases with time. This means that heat transported with the polymer by convection is much smaller than the amount of heat conducted through the polymer.

\subsection{Inertial forces}

In conventional injection molding simulations it is common to assume Stokes flow conditions. This means that advective inertial forces are neglected because they are dominated by viscous forces. The ratio between inertial effects to viscous forces can be characterized by the Reynolds number

$$
\operatorname{Re}=\frac{\rho \hat{U} \hat{L}}{\hat{\eta}}
$$

On the microscale, both the characteristic length and characteristic velocity are smaller than on the macroscale. At the start of the simulations the Reynolds number is below $10^{-6}$ and, as the viscosity rapidly increases, the Reynolds number is further reduced. Hence, the assumption of Stokes flow is still valid on the microscale.

\subsection{Wall slip}

For polyolefins it has been observed that the critical shear stress required for cohesive failure and onset of wall slip scales linearly with the adhesion energy. For the interface between HDPE and steel the critical value is $0.1-0.18 \mathrm{MPa}$. (Anastasiadis and Hatzikiriakos, 1998) The maximum shear stress in the present simulations exceeds this, with wall shear stresses up to $1 \mathrm{MPa}$ during the filling phase. It is therefore likely that flow instabilities can occur. However, even if the polymer would slip at the wall, there will still be zero velocity at the sharp lower corners of the grating (where the mold wall tangents $y=0$ in Fig. 4) and it is unlikely that wall slip will have a large influence on the replication.

\subsection{Elastic effects due to fountain flow}

In injection molding there is a fountain flow region close to the flow front. In this region, melt will be transported from the midplane of the flow field and out towards the wall. In this fountain flow, the polymer will be stretched and sheared causing high molecular orientation and a state of tension close to the mold wall.

In the paper by Eriksson et al, it is argued that elastic effects in the flow front region influence the filling of microfeatures. They define a Deborah number De as the ratio of storage modulus $G$ ' to loss modulus $G$ ', at a frequency equal to the macroscopic flow front velocity $v_{\text {macro }}$ divided by the half thickness of the geometry $H$.

$$
\mathrm{De}=\frac{G^{\prime}\left(v_{\text {macro }} / H\right)}{G^{\prime \prime}\left(v_{\text {macro }} / H\right)}
$$

For Deborah numbers above 0.1 , they observe improved replication at isothermal conditions. In the present simulations, the Deborah number as defined by Eq. 28 is between 0.5 and 1.2. The moduli are then evaluated at $270{ }^{\circ} \mathrm{C}$ using data from the dynamic rheology measurements. The De values indicate that elastic effects might influence the filling also in our case.

In order to reduce De to below 0.1 , it would be necessary to either reduce the injection velocity to $0.01 \mathrm{~m} / \mathrm{s}$ while keeping the melt temperature at $270{ }^{\circ} \mathrm{C}$ or keep the injection velocity at $0.25 \mathrm{~m} / \mathrm{s}$ and increase the melt temperature to $410{ }^{\circ} \mathrm{C}$.

\subsection{Heating of the mold wall}

As seen in Fig. 10, the temperature of the mold surface increases significantly as the polymer comes in contact with it. The effusivity based contact temperature is a reasonable estimate for the mold wall temperature at times when good thermal contact between the two materials has been established. The estimate is better for high injection velocities since a better thermal contact between the melt and the wall is established earlier.

However, within the timescale of the microfeature filling, the contact temperature is lower than that based 
on an effusivity approach, due to the finite heat transfer coefficient. At longer times $(>0.5 \mathrm{~s})$ the $\mathrm{BeCu}$ block under the nickel shim is heated and its higher thermal diffusivity reduces the contact temperature from what would be expected from an effusivity based argument.

Nevertheless, an effusivity based contact temperature is in better agreement with the simulated results in this study than using a constant mold temperature. It can therefore be recommended as a first guess for the mold temperature increase.

The relatively small increase in the surface temperature of the mold is usually neglected in commercial injection molding simulation software. But, as can be seen in Fig. 9 , a $5^{\circ} \mathrm{C}$ increase in the mold temperature has a significant effect on the replication, both in the simulations and experimentally, and this effect has to be included for a realistic melt temperature evolution.

\subsection{Rheology close to the glass transition}

As seen in Fig. 2, the rheology close to the glass transition is not well described by a Cross-WLF model. Also, as the temperature decreases the elastic contribution to the rheological response will increase. This effect is not included in our simulations, and it is common to neglect this effect in conventional injection molding simulations. We see, however, in Fig. 7 that a significant part of the deformation of the polymer into the microfeature occurs at temperatures close to the glass transition. A more realistic description of how microfeatures are replicated in injection molding may need a viscoelastic model. However, viscoelastic simulations require special computational codes and more detailed polymer characterization. Furthermore, many viscoelastic simulations have mainly been demonstrated for isothermal flow.

\section{Conclusions}

Simulations predicting how the replication of microfeatures changes with injection molding processing settings have been demonstrated.

The size of the microfeatures is in a transition region where several physical effects which normally are disregarded in injection molding start becoming important. One such effect is the wall adhesion, which can have a larger effect on the filling of microfeatures than the cavity pressure the first millisecond after polymer wall contact. It is also observed that the microfeatures partly fill at temperatures close to the glass transition. At such low temperatures, the assumption of viscous flow gradually becomes worse. Nevertheless, simplified viscous simulations are able to capture how changes in the processing settings influence the replication.

The high sensitivity of replication to changes in mold temperature is predicted by the simulations. To be able to realistically capture this temperature sensitivity, it was necessary to include the heating of the mold wall explicitly in the simulations.

\section{Appendix}

To reconstruct the degree of replication as defined in the experimental paper (Tofteberg et al., 2008), the following procedure was used;

1. Find the shape of a single period $y(x)$ of the grating from 0 to $x=L_{\mu}=3 \mu \mathrm{m}$. This shape follows the mold wall where there is contact between polymer and wall and the contour line $r_{\mathrm{COC}}=0.5$ elsewhere.

$$
y(x)=h(x) \quad \forall x \in\left\lfloor 0, L_{\mu}\right\rfloor
$$

2. Shift this period along the $x$-axis and repeat the shape 15 times. The value 15 was chosen to match the region investigated in the experimental study.

$$
y(x)=y\left(x-L_{\mu}\right) \quad \forall x \in\left\lfloor L_{\mu}, 15 L_{\mu}\right\rfloor
$$

3. Calculate the power spectrum density (PSD) of this grating. This was done numerically using the implementation of the fast Fourier transform in Matlab (Matlab R2007a, 2007).

$$
\Phi(\omega)=\left|\frac{1}{\sqrt{2 \pi}} \int_{0}^{15 L_{\mu}} y(x) e^{-i \omega x} \mathrm{~d} x\right|^{2}
$$

4. Integrate the main peak of the PSD and divide by the integral of the PSD peak of the mold to obtain the degree of replication DOR. The half width of the integration $\Delta \omega$ is chosen so that both the local minima on each side of the peak are included in the integration.

$$
D O R=\sqrt{\frac{\int_{1 / L_{\mu}-\Delta \omega}^{1 / L_{\mu}-\Delta \omega}}{\int_{L_{\mu}+\Delta \omega}^{1 / L_{\mu}+\Delta \omega} \Phi_{\text {mold }}(\omega) \mathrm{d} \omega}}
$$

\section{References}

Anastasiadis, S. H. and Hatzikiriakos, S. G., "The work of adhesion of polymer/wall interfaces and its association with the onset of wall slip", J. Rheol., 42, 795-812 (1998)

ANSYS CFX-11.0 from ANSYS Europe Ltd. (2007)

Baehr, H. D. and Stephan, K.: Wärme- und

Stoffübertragung, 4th ed., Springer, Heidelberg (2004)

Blochowiak, M., et al., "Thermodynamics and rheology of cycloolefin copolymers", J. Chem. Phys., 124, art no 134903 (2006)

Eriksson, T. and Rasmussen, H. K., "The effects of polymer melt rheology on the replication of surface microstructures in isothermal moulding", J. NonNewtonian Fluid Mech., 127, 191-200 (2005) 
Ferry, J. D.: Viscoelastic properties of polymers, 3rd ed., John Wiley \& Sons, Inc., New York (1980)

Gadegaard, N., et al., "Biomimetic polymer nanostructures by injection molding", Macromol. Mater. Eng., 288, 76-83 (2003)

Gramberg, H. J., et al., "Flow patterns behind the free flow front for a Newtonian fluid injected between two infinite parallel plates", Eur. J. Mech. B-Fluid., 23, 571-585 (2004)

Khanarian, G. and Celanese, H., "Optical properties of cyclic olefin copolymers", Opt. Eng., 40, 1024-1029 (2001)

Kim, S. W. and Turng, L. S., "Three-dimensional numerical simulation of injection molding filling of optical lens and multiscale geometry using finite element method", Polym. Eng. Sci., 46, 1263-1274 (2006)

Kim, Y., et al., "Modelling of passive heating for replication of sub-micron patterns in optical disk substrates", J. Phys. D: Appl. Phys., 37, 1319-1326 (2004)

Matlab R2007a from The MathWorks (2007)

Moldflow Plastics Insight (MPI) 6.1 from Moldflow Corporation (2007)

Puukilainen, E., et al., "Superhydrophobic polyolefin surfaces: Controlled micro- and nanostructures", Langmuir, 23, 7263-7268 (2007)

Shin, J. Y., et al., "Chemical structure and physical properties of cyclic olefin copolymers - (IUPAC technical report)", Pure Appl. Chem., 77, 801-814 (2005)

Saarikoski, I., et al., "Modification of polycarbonate surface properties by nano-, micro-, and hierarchical micro-nanostructuring", Appl. Surf. Sci., 255, 90009005 (2009)

Tofteberg, T., et al., "Injection molding of a diffractive optical element", Polym. Eng. Sci., 48, 2134-2142 (2008)

Vahlund, C. F., "Using a finite volume approach to simulate the mould filling in compression moulding", J. Reinf. Plast. Compos., 22, 499-515 (2003)

Yao, D., et al., "Rapid thermal cycling of injection molds: An overview on technical approaches and applications", Adv. Polym. Technol., 27, 233-255 (2008)

Yao, D. G. and Kim, B., "Scaling issues in miniaturization of injection molded parts", J. Manuf. Sci. E.-T. ASME, 126, 733-739 (2004)

Yoshii, M., et al., "Experimental study of the transcription of minute width grooves by injection molding (II)", Polym. Eng. Sci., 38, 1587-1593 (1998)

Young, W. B., "Analysis of filling distance in cylindrical microfeatures for microinjection molding", Appl. Math. Model., 31, 1798-1806 (2007)

$\mathrm{Yu}$, L., et al., "Flow and heat transfer simulation of injection molding with microstructures", Polym. Eng. Sci., 44, 1866-1876 (2004) 